\title{
Evaluation of an initiative to provide omega-3 rich snacks to preschool children in Edinburgh
}

\author{
E. A. D. Scheers Andersson, S. Drummond and J. M. McKenzie \\ Queen Margaret University, Edinburgh EH21 6UU, Scotland, UK
}

The benefits of the long-chain omega-3 PUFA are widely documented, particularly concerning optimising childhood development and reducing the risk of CVD. Synthesis of the LC $n$-3 PUFA from the parent fatty acid, $\alpha$-linolenic acid, is limited, despite $\alpha$-linolenic acid being abundant in the British diet. Dietary preformed LC $n-3$ PUFA are found predominantly in oily fish, which is less frequently consumed.

According to the recently published National Diet and Nutrition Survey (NDNS 2008/2009) ${ }^{(1)}$, the majority of preschool children in Britain have inadequate intakes of oily fish and subsequently LC $n-3$ PUFA. Various factors such as strong taste and smell, and adult preconceptions about acceptance, impede the introduction of oily fish into the diet at a young age. Overcoming these barriers, however, will increase the likelihood of adopting lifelong healthy eating behaviours.

An initiative was developed and set-up in Olivebank Child and Family centre in East Lothian in order to provide a range of snack foods containing oily fish and consequently increase intakes of LC $n-3$ PUFA in preschool children. The aim of this study was to evaluate the uptake and acceptability of the novel fish-snacks compared with the regular snacks, to assess the nutritional content per portion and per child's intake of the two snack groups, and to attain feedback from the nursery staff by means of structured interviews.

Over a period of $16 \mathrm{~d}$, fourteen preschool children (64\% male and $36 \%$ female) were recruited with a mean age of 3.4 years (range, 3-4 years). The four different oil-rich snacks were presented in the afternoons, approximately $2 \mathrm{~h}$ after lunch time. There was no significant difference in uptake of the two snack groups $(P>0.05)$. Acceptability of the snacks, assessed using a pictorial Likert scale, was also comparable with no significant difference between groups $(P>0.05)$. The omega-3 rich snacks (which included smoked mackerel pâté, smoked mackerel and spinach tartlets, salmon fish fingers and tuna meatballs) provided a significantly higher mean amount of EPA and DHA per portion $(191 \mathrm{mg})(P<0.001)$ and per child's intake $(136 \mathrm{mg})(P<0.001)$ in comparison with the regular snacks that did not contain any LC $n$-3 PUFA. The feedback from staff interviews demonstrated their understanding of the benefits of the oil-rich fish snacks to the children, and they expressed their confidence in preparing the snacks and their willingness to carry on with the initiative.

In conclusion, the majority of children found the range of omega-3 rich snacks as enjoyable and acceptable as the regular snacks. The impact of this initiative on the children's overall intake of LC $n-3$ PUFA requires further evaluation; however, the results from this study indicate that such an initiative can be successful within a similar vulnerable population.

Funding was provided by the Organix Foundation.

1. National Diet and Nutrition Survey. Headline Results from Year 1 of the Rolling Programme. 2008/2009. London: Food Standards Agency and the Department of Health. 